\title{
Multivariate Analysis of Relationship between Childhood Trauma and Psychotic Symptoms in Patients with Schizophrenia
}

\author{
Sungun Chae', Minyoung $\mathrm{Sim}^{2,3 凶}{ }^{1}$, Mijeong $\mathrm{Lim}^{1}$, Joonho $\mathrm{Na}^{4}$, and Daeho $\mathrm{Kim}^{5}$ \\ 1'Department of Psychiatry, Seoul National Hospital, Seoul, Republic of Korea \\ ${ }^{2}$ Department of Psychiatric Rehabilitation, Seoul National Hospital, Seoul, Republic of Korea \\ ${ }^{3}$ Psychological Trauma Center, Seoul National Hospital, Seoul, Republic of Korea \\ ${ }^{4}$ Department of Emergency Medicine, Asan Chungmu Hospital, Asan, Republic of Korea \\ ${ }^{5}$ Department of Psychiatry, Hanyang University Medical School, Seoul, Republic of Korea
}

\begin{abstract}
The aim of this study was to examine the relationship between childhood trauma and psychotic symptoms in schizophrenic patients after controlling for the possible confounding factors, such as depression and dissociative symptoms. Ninety-eight schizophrenic inpatients participated. Childhood trauma was examined using the Childhood Trauma Questionnaires (CTQ), which consists of physical abuse (PA), sexual abuse (SA), emotional abuse (EA), physical neglect (PN), and emotional neglect (EN). Positive and Negative Syndrome Scale (PANSS), Dissociative Experience Scale (DES), and Beck's Depression Inventory (BDI) were also administered. Data were analyzed by partial correlation and general linear model. The total score of CTQ was positively correlated with positive, general, and total scores of PANSS. All five types of childhood trauma were associated with dissociative symptoms. EA and EN were positively correlated with depressive symptoms. Only SA significantly predicted positive symptoms of schizophrenia after controlling for age, sex, BDI, and DES scores, with a dose-response relationship between SA and positive symptoms.

Psychiatry Investig 2015;12(3):397-401
\end{abstract}

Key Words Childhood trauma, Positive symptom, Sexual abuse, Schizophrenia.

\section{INTRODUCTION}

Increased prevalence and long-term lingering effects of childhood trauma are seen in individuals with psychotic disorders, including schizophrenia. Rates of childhood sexual or physical abuse among patients with schizophrenia are substantial; one study concluded that $21-65 \%$ of individuals with schizophrenia had such experiences. ${ }^{1}$ Patients with increased experience of childhood trauma demonstrated worse mental and physical health, poorer social function, and non-adherence or lower treatment engagement during the course of their schizophrenic illness than those with less or no childhood trauma experience. ${ }^{2,3}$

Relationship between psychotic symptoms and childhood trauma has also been explored. Overall, childhood abuse cor-

Received: August 14, 2014 Revised: October 18, 2014

Accepted: October 23, 2014 Available online: July 6, 2015

$\triangle$ Correspondence: Minyoung Sim, MD, PhD

Department of Psychiatry, Psychological Trauma Center, Seoul National Hospital, 398 Neungdong-ro, Gwangjin-gu, Seoul 143-711, Republic of Korea Tel: +82-2-2204-0327, Fax: +82-2-2204-0394

E-mail: minyoung.sim.yb@gmail.com

(c) This is an Open Access article distributed under the terms of the Creative Commons Attribution Non-Commercial License (http://creativecommons.org/licenses/by$\mathrm{nc} / 3.0$ ) which permits unrestricted non-commercial use, distribution, and reproduction in any medium, provided the original work is properly cited. related with positive symptoms of schizophrenia.-8 However, this was not replicated in at least two non-North American studies. ${ }^{9,10}$ Findings for negative symptoms are mixed; some studies found a significant positive correlation between negative symptoms $s^{11,12}$ and past trauma, while others reported opposite finding (i.e., fewer negative symptoms). ${ }^{8}$ These symptomatic response may differ according to the type of childhood trauma, for example, childhood abuse was associated with positive symptoms while childhood neglect was associated with negative symptoms. ${ }^{11}$

Relationship between past trauma and current schizophrenic symptoms is far more complex than previously thought: studies have suggested that the impact of childhood trauma on psychotic symptoms is mediated by dissociation ${ }^{13,14}$ and depressive symptoms. ${ }^{15,16}$ Dissociation is also strongly associated with childhood trauma in general and this link has been well replicated in schizophrenia. ${ }^{13,17}$ Dissociation may overlap with positive psychotic symptoms, especially in the acute period, thus confounding the relationship between childhood trauma and positive symptoms. ${ }^{13}$ One study showed that dissociation has more to do with schizophrenic symptoms, including positive and negative symptoms, than previous trauma. ${ }^{14}$ Depressive symptoms are also associated with childhood 
trauma, especially emotional abuse and neglect, in schizophrenia. ${ }^{16}$ Depression also confounds and overlaps with negative symptoms of schizophrenia. ${ }^{18}$ In addition, one general population study in the United Kingdom showed that depression mediated the association between childhood sexual abuse and psychosis. $^{15}$

No previous studies included and controlled for both dissociation and depression as symptomatic confounders when investigating the association between childhood trauma and schizophrenic symptoms. Therefore, we examined a convenience sample of schizophrenic inpatients at a psychiatric hospital to confirm the relationship between childhood trauma experiences and psychotic symptoms after controlling for the possible confounder variables, dissociative and depressive symptoms. We also evaluated which types of childhood trauma experiences are associated with psychotic symptom dimensions.

\section{METHODS}

\section{Participants}

All participants were recruited from the psychiatric inpatient units at Seoul National Hospital, South Korea, using convenience sampling over a two-year period. Patients were considered eligible to enter the study if they had a DSM-IV-TR ${ }^{19}$ diagnosis of schizophrenia, illness duration for at least one year with stable and consistent diagnostic features, were between 18-65 years of age, and their charging psychiatrist was in favor of their participation in the study. The duration of illness was a mean of 19.4 years $(\mathrm{SD}=8.9)$ and all participants were receiving at least one type of antipsychotic medication. Written informed consent was obtained from each patient after the institutional review board approval.

A total of 105 patients participated in the survey; however, data from seven patients were excluded due to incomplete questionnaires or lack of interview-based data, leaving a final sample of 98. Demographic and clinical characteristics of participants are provided in Table 1 .

\footnotetext{
Assessment

Psychotic symptoms were evaluated using the Positive and Negative Syndrome Scale (PANSS), 20 which consists of three subscales (positive, negative, and general psychopathology) with a total of 30 items. Childhood trauma was assessed with the Childhood Trauma Questionnaire-Short Form (CTQ-SF), ${ }^{21}$ a retrospective 28 -item self-report instrument developed to evaluate childhood trauma experiences. CTQ-SF evaluates three abuse experiences [i.e., emotional abuse (EA), physical abuse (PA), and sexual abuse (SA)] and two neglect experiences [i.e., emotional neglect (EN) and physical neglect (PN)].
}

Table 1. Demographic and clinical characteristics of subjects $(\mathrm{N}=$ 98)

\begin{tabular}{lc}
\hline \multicolumn{1}{c}{ Variables } & N (\%) or mean \pm SD \\
\hline Gender (males) & $51(52)$ \\
Age (years) & $43.0 \pm 9.4$ \\
Education level (years) & $12.4 \pm 3.1$ \\
Current marital status & \\
Married or cohabiting & $7(7.1)$ \\
Divorced or separated & $15(15.3)$ \\
Widowed & $1(1.0)$ \\
Single & $73(74.5)$ \\
Unknown & $2(2.0)$ \\
Duration of illness (years) & $19.4 \pm 8.9$ \\
Numbers of previous hospitalization & $7.5 \pm 6.2$ \\
Positive and Negative Syndrome Scale & \\
Positive subscales & $16.2 \pm 6.1$ \\
Negative subscales & $17.1 \pm 7.1$ \\
General subscales & $32.4 \pm 6.3$ \\
Total scores & $65.7 \pm 15.2$ \\
Childhood trauma questionnaires & \\
Emotional abuse & $13.3 \pm 4.9$ \\
Physical abuse & $10.4 \pm 3.3$ \\
Sexual abuse & $48.9 \pm 14.1$ \\
Emotional neglect & $18.9 \pm 17.5$ \\
Physical neglect & $13.9 \pm 12.3$ \\
Total scores & $8.0 \pm 3.5$ \\
Dissociative Experience Scale & $7.8 \pm 3.7$ \\
\hline & \\
&
\end{tabular}

Three dimensional scores-emotional (EA+EN), physical (PA+ $\mathrm{PN}$ ), and sexual (SA) experiences-were also calculated. Dissociative symptoms were covered by the Dissociative Experience Scale (DES), 22 a 28-item self-report measure for assessment of dissociative phenomena in daily life. The Beck Depression Inventory (BDI) ${ }^{23}$ a self-report questionnaire consisting of 21 items, was administered to measure the severity of depression. Validation data for the Korean versions of the symptom inventories used in this study are available. ${ }^{24-27}$

\section{Statistical analysis}

The relationships among childhood trauma experiences and depression, dissociation, and psychotic symptoms in schizophrenic patients were evaluated by partial correlation analysis after controlling for age and sex effects. General linear regression analysis was used to evaluate the contribution of childhood trauma experiences to psychotic symptoms. Statistical significance was defined as an alpha value of $<0.05$ (twotailed). All statistical analyses were performed using Statistica 
software (version 7.0).

\section{RESULTS}

\section{Childhood trauma and symptoms}

CTQ-SF total score was positively correlated with PANSS positive, general, and total scores $(\mathrm{p}=0.014, \mathrm{p}<0.001, \mathrm{p}=0.002$, respectively). Within the subscales of CTQ-SF, all of the abuse (EA, PA, and SA) scores were positively correlated with PANSS total score $(p=0.033, p=0.034$ and $p<0.001$, respectively), and sexual abuse (SA) score was positively correlated with PANSS positive score ( $\mathrm{p}=0.001$ ) (Table 2, Figure 1A). Positive symptom score was predicted by sexual abuse and dissociation after controlling for sex and age effects (Table 3). Other

Table 2. Partial correlations for CTQ, PANSS, DES, and BDI scores

\begin{tabular}{|c|c|c|c|c|c|c|c|c|c|}
\hline \multirow{3}{*}{ CTQ } & \multicolumn{7}{|c|}{ PANSS } & \multirow{3}{*}{ DES } & \multirow{3}{*}{ BDI } \\
\hline & \multicolumn{4}{|c|}{ Positive } & \multirow{2}{*}{ Negative } & \multirow{2}{*}{ General } & \multirow{2}{*}{ Total } & & \\
\hline & Delusion & Disorganization & Hallucination & Total & & & & & \\
\hline EA & 0.06 & 0.13 & 0.17 & 0.15 & 0.06 & $0.35^{*}$ & $0.22 *$ & $0.47^{*}$ & $0.29^{*}$ \\
\hline PA & 0.10 & 0.17 & $0.21^{*}$ & 0.21 & 0.07 & $0.28 *$ & $0.22 *$ & $0.36^{*}$ & 0.12 \\
\hline SA & $0.22 *$ & $0.29 *$ & $0.24^{*}$ & $0.33^{*}$ & 0.13 & $0.46^{*}$ & $0.37^{*}$ & $0.50^{*}$ & 017 \\
\hline EN & 0.15 & $0.21^{*}$ & 0.16 & 0.12 & 0.10 & $0.21^{*}$ & 0.18 & $0.23^{*}$ & $0.29^{*}$ \\
\hline $\mathrm{PN}$ & 0.07 & 0.20 & 0.18 & 0.12 & 0.15 & 0.02 & 0.13 & $0.35^{*}$ & 0.21 \\
\hline Total & 0.17 & $0.28 *$ & $0.27^{*}$ & $0.26^{*}$ & 0.14 & $0.38^{*}$ & $0.32 *$ & $0.53^{*}$ & $0.32^{*}$ \\
\hline
\end{tabular}

All values are Pearson's r. after controlling for age and sex. * p-value $<0.05$. CTQ: Childhood trauma questionnaires, PANSS: Positive and Negative Syndrome Scale, DES: Dissociative Experience Scale, BDI: Beck's Depression Inventory, EA: emotional abuse, PA: physical abuse, SA: sexual abuse, EN: emotional neglect, PN: physical neglect

Table 3. General linear model for PANSS positive subscale scores

\begin{tabular}{lrrrc}
\hline \multicolumn{1}{c}{ Variables } & SS & MS & F & 0.34 \\
\hline Age & 10.61 & 10.61 & 0.67 & 0.559 \\
Beck's Depression Inventory & 20.7 & 20.7 & 4.34 & 0.414 \\
Dissociative Experience Scale* & 133.51 & 133.51 & & 0.040 \\
CTQ subscale & & & 2.65 & 0.107 \\
Emotional abuse & 81.64 & 81.63 & 0.18 & 0.675 \\
Physical abuse & 5.45 & 5.45 & 5.16 & 0.026 \\
Sexual abuse* & 158.90 & 158.90 & 0.22 & 0.641 \\
Emotional neglect & 6.74 & 6.74 & 0.10 & 0.758 \\
Physical neglect & 2.95 & 2.95 & & \\
\hline
\end{tabular}

*p-value<0.05. PANSS: Positive and Negative Syndrome Scale, CTQ: childhood trauma questionnaire, SS: sum of squares, MS: means squares
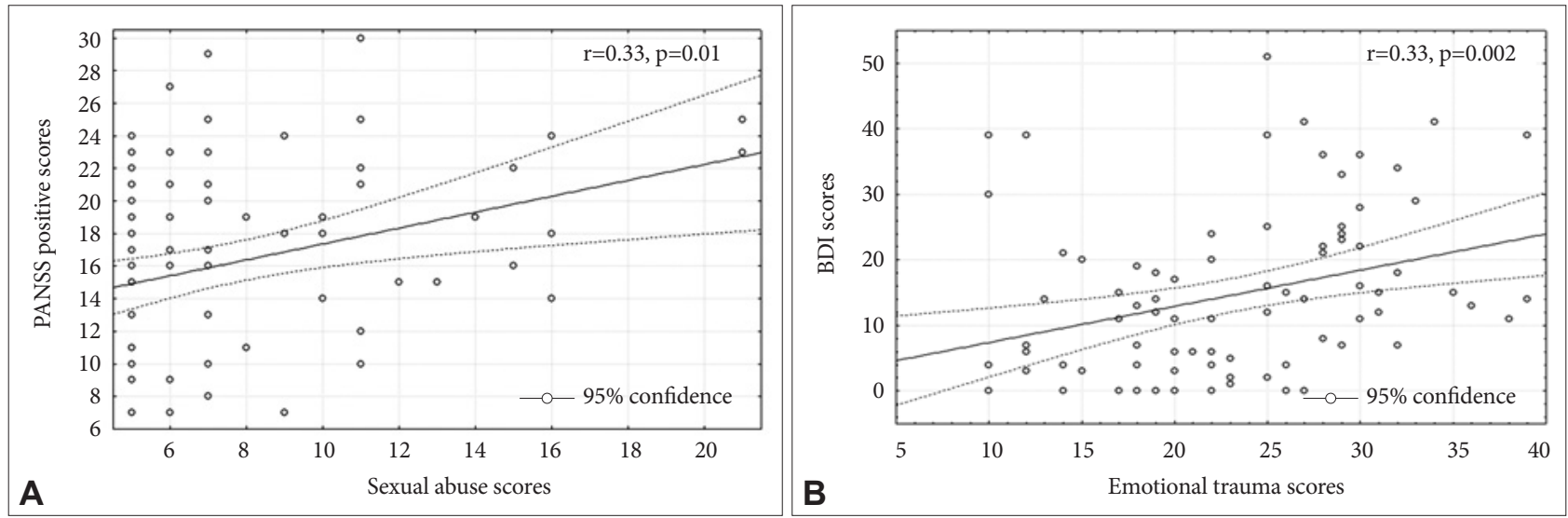

Figure 1. Relationship between childhood trauma and psychopathology. A: Sexual abuse (SA) scores were positively correlated with PANSS positive scores. B: Emotional trauma scores (EA+EN) were positively correlated with depressive symptom scores. PANSS: Positive and Negative Syndrome Scale, EA: emotional abuse, EN: emotional neglect. 
types of childhood trauma showed no significant contribution in general linear regression analysis. All types of childhood trauma were associated with DES scores $(\mathrm{p}<0.001-0.033)$ and emotional abuse and neglect were related to BDI scores ( $\mathrm{p}=$ 0.006 and $p=0.005$, respectively) (Figure $1 \mathrm{~B}$ ).

\section{DISCUSSION}

We investigated an inpatient sample of schizophrenia to confirm that there was a link between childhood trauma and psychotic symptoms even after controlling for confounding variables. In this study, sexual abuse in childhood was a significant predictor of positive symptoms after controlling for dissociation and depression. Dissociation also remained a significant predictor indicating that it independently contributes to positive symptoms of schizophrenia.

Our finding of a link between child sexual abuse and positive symptoms is consistent with previous studies that performed bivariate analyses of treatment-seeking first-onset schizophrenia ${ }^{28}$ and adult psychosis in the community. ${ }^{15}$ However, our study further proved that even after controlling for dissociation and depressive symptoms, child sexual abuse was associated with positive symptoms. This is in contrast to previous findings that association of childhood trauma with schizophrenic symptoms is confounded by dissociation ${ }^{17}$ or that dissociation is more closely related to positive symptoms than traumatic events, thus emphasizing the role of dissociation in relation to schizophrenic symptoms. ${ }^{14}$ Our finding is more appealing in that through multivariate analysis, we found that both dissociation and childhood sexual abuse were independent predictors of positive symptoms.

Among positive symptoms, several studies have noted that only hallucination was linked to child abuse. ${ }^{5}$ Our data also showed a significant association between hallucination and SA. However, other positive symptoms such as delusion and disorganization also showed a significant relationship with SA.

Another interesting finding was the relationship between EA/EN and depressive symptoms. This specific connection has been documented in primary care patients, ${ }^{30}$ outpatients with major depression, ${ }^{31}$ and patients with first-onset schizophrenia. ${ }^{16}$ Thus, it is likely that this relationship also exists in inpatients with more advanced schizophrenic illness.

We acknowledge some limitations to this study. This study relied on retrospective self-reports of childhood trauma, and so the accuracy of these reports may be in question, especially considering that the study involved schizophrenic patients. ${ }^{13}$ However, a previous study reported that retrospective reporting of childhood abuse by psychosis patients is typically reliable when judged against siblings, stable over long periods, and unaffected by current symptoms. ${ }^{32}$ Second, this study fo- cused only on childhood traumatic events and their interactions or the effects of adulthood trauma were not considered. This may limit our findings in that one study noted that persistence of positive symptoms in older adults with schizophrenia was related only to lifetime trauma, not childhood trauma. ${ }^{33}$

The strengths of this study include our examination of a homogenous group with the exclusive diagnosis of schizophrenia, an adequate sample size, and multivariate analysis controlling for confounding variables. Further studies may benefit from targeting more diverse groups of schizophrenia patients and investigating life-time traumas and adversities. Association with specific types of schizophrenic symptoms may provide more insight into the effect of trauma in the presentation of schizophrenic illness. Consequently, greater attention should be given to trauma history among individuals with schizophrenia, and clinicians and psychiatric staffs should be professionally trained to assess trauma history and its impacts to enable them to formulate more comprehensive and appropriate treatment plans for such patients.

\section{Acknowledgments}

This study was supported by a grant (2013-05) from the Seoul National Hospital, Republic of Korea.

\section{REFERENCES}

1. Read J, Findk PJ, Rudegeair T, Felitti B, Whitfield CL. Child maltreatment and psychosis: a return to a genuinely integrated bio-psycho-social model. Clin Schizophr Relat Psychoses 2008;2:235-254.

2. Lecomte T, Spidel A, Leclerc C, MacEwan GW, Greaves C, Bentall RP. Predictors and profiles of treatment non-adherence and engagement in services problems in early psychosis. Schizophr Res 2008;102:295-302.

3. Rosenberg SD, Lu W, Mueser KT, Jankowski MK, Cournos F. Correlates of adverse childhood events among adults with schizophrenia spectrum disorders. Psychiatr Serv 2007;58:245-253.

4. Burns JK, Jhazbhay K, Esterhuizen T, Emsley R. Exposure to trauma and the clinical presentation of first-episode psychosis in South Africa. J Psychiatr Res 2011;45:179-184.

5. Kilcommons AM, Morrison AP. Relationships between trauma and psychosis: an exploration of cognitive and dissociative factors. Acta Psychiatr Scand 2005;112:351-359.

6. Lysaker PH, Larocco VA. The prevalence and correlates of trauma-related symptoms in schizophrenia spectrum disorder. Compr Psychiatry 2008;49:330-334.

7. Ross CA, Anderson G, Clark P. Childhood abuse and the positive symptoms of schizophrenia. Hosp Community Psychiatry 1994;45:489491.

8. Wang Z, Xue Z, Pu W, Yang B, Li L, Yi W, et al. Comparison of first-episode and chronic patients diagnosed with schizophrenia: symptoms and childhood trauma. Early Interv Psychiatry 2013;7:23-30.

9. Amr M, El-Wasify M, Amin T, Roy A. Childhood trauma in Egyptian patients with schizophrenia: a cry over Nile. Schizophr Res 2010;124: 242-243.

10. Kim D, Kaspar V, Noh S, Nam JH. Sexual and physical abuse among Korean female inpatients with schizophrenia. J Trauma Stress 2006; 19:279-289.

11. Gallagher BJ 3rd, Jones BJ. Childhood stressors and symptoms of schizophrenia. Clin Schizophr Relat Psychoses 2013;7:124-130.

12. Vogel M, Meier J, Gronke S, Waage M, Schneider W, Freyberger HJ, et 
al. Differential effects of childhood abuse and neglect: mediation by posttraumatic distress in neurotic disorder and negative symptoms in schizophrenia? Psychiatry Res 2011;189:121-127.

13. Schafer I, Fisher HL, Aderhold V, Huber B, Hoffmann-Langer L, Golks $\mathrm{D}$, et al. Dissociative symptoms in patients with schizophrenia: relationships with childhood trauma and psychotic symptoms. Compr Psychiatry 2012;53:364-371.

14. Vogel M, Schatz D, Spitzer C, Kuwert P, Moller B, Freyberger HJ, et al. A more proximal impact of dissociation than of trauma and posttraumatic stress disorder on schneiderian symptoms in patients diagnosed with schizophrenia. Compr Psychiatry 2009;50:128-134.

15. Bebbington P, Jonas S, Kuipers E, King M, Cooper C, Brugha T, et al. Childhood sexual abuse and psychosis: data from a cross-sectional national psychiatric survey in England. Br J Psychiatry 2011;199:29-37.

16. Sahin S, Yuksel C, Guler J, Karadayi G, Akturan E, Gode E, et al. The history of childhood trauma among individuals with ultra high risk for psychosis is as common as among patients with first-episode schizophrenia. Early Interv Psychiatry 2013;7:414-420.

17. Sar V, Taycan O, Bolat N, Ozmen M, Duran A, Ozturk E, et al. Childhood trauma and dissociation in schizophrenia. Psychopathology 2010; 43:33-40.

18. Chemerinski E, Bowie C, Anderson H, Harvey PD. Depression in schizophrenia: methodological artifact or distinct feature of the illness? J Neuropsychiatry Clin Neurosci 2008;20:431-440.

19. American Psychiatric Association. Task Force on DSM-IV. Diagnostic and statistical manual of mental disorders DSM-IV-TR. Washington, DC: American Psychiatric Association; 2000.

20. Kay SR, Fiszbein A, Opler LA. The positive and negative syndrome scale (PANSS) for schizophrenia. Schizophr Bull 1987;13:261-276.

21. Bernstein DP, Stein JA, Newcomb MD, Walker E, Pogge D, Ahluvalia T, et al. Development and validation of a brief screening version of the Childhood Trauma Questionnaire. Child Abuse Negl 2003;27:169-190.

22. Bernstein EM, Putnam FW. Development, reliability, and validity of a dissociation scale. J Nerv Ment Dis 1986;174:727-735.

23. Beck AT, Ward CH, Mendelson M, Mock J, Erbaugh J. An inventory for measuring depression. Arch Gen Psychiatry 1961;4:561-571.

24. Yi JS, Ahn YM, Shin HK, An SK, Joo YH, Kim SH, et al. Reliability and validity of the Korean version of the positive and negative syndrome scale. J Korean Neuropsychiatr Assoc 2001;40:1090-1105.

25. Kim D, Park SC, Yang H, Oh DH. Reliability and validity of the Korean version of the childhood trauma questionnaire-short form for psychiatric outpatients. Psychiatry Investig 2011;8:305-311.

26. Park JM, Choe BM, Kim MJ, Hahn HM, Yoo SY, Kim SH, et al. Standardization of dissociative experiences scale-Korean version. Korean J Psychopathol 1995;4:105-125.

27. Lee YH, Song JY. A study of the reliability and the validity of the BDI, SDS, and MMPI-D scales. Korean J Clin Psychol 1991;10:98-113.

28. Ucok A, Bikmaz S. The effects of childhood trauma in patients with first-episode schizophrenia. Acta Psychiatr Scand 2007;116:371-377.

29. Daalman K, Diederen KM, Derks EM, van Lutterveld R, Kahn RS, Sommer IE. Childhood trauma and auditory verbal hallucinations. Psychol Med 2012;42:2475-2484.

30. Spertus IL, Yehuda R, Wong CM, Halligan S, Seremetis SV. Childhood emotional abuse and neglect as predictors of psychological and physical symptoms in women presenting to a primary care practice. Child Abuse Negl 2003;27:1247-1258.

31. Bernet CZ, Stein MB. Relationship of childhood maltreatment to the onset and course of major depression in adulthood. Depress Anxiety 1999;9:169-174.

32. Fisher HL, Craig TK, Fearon P, Morgan K, Dazzan P, Lappin J, et al. Reliability and comparability of psychosis patients' retrospective reports of childhood abuse. Schizophr Bull 2011;37:546-553.

33. Cohen CI, Palekar N, Barker J, Ramirez PM. The relationship between trauma and clinical outcome variables among older adults with schizophrenia spectrum disorders. Am J Geriatr Psychiatry 2012;20:408-415. 